\title{
Launch abort trajectory optimisation for reusable launch vehicles
}

\author{
Federico Toso* and Christie Alisa Maddock ${ }^{\dagger}$ \\ Centre for Future Air Space Transportation Technologies \\ University of Strathclyde, Glasgow, G1 1XJ, United Kingdom
}

\begin{abstract}
Among the future space access vehicles, the lifting body spaceplane is the most promising approach to prevent damage to both the launcher and the payload in case of loss of thrust. The glide performances of the vehicle allow the recovery in both nominal and abort cases. The approach presented is used in the investigation of the unpowered descent paths of a sample vehicle through trajectory optimisation. The vehicle's downrange and crossrange limits are obtained for aborts in multiple flight conditions.
\end{abstract}

\section{Introduction}

The main approach to deliver payloads into space has historically been the expendable vertically launched rocket. Recent advancements towards the partial or full re-usability of the first stages holds the potential to significantly lower the cost of access to space and open a broader market. One reusable launcher that could emerge in this market is the spaceplane, a lifting body vehicle can reduce the aerodynamic losses of the ascent and take advantage of effective air-breathing engines that do not require on-board storage of the oxidizer. Those hybrid or combined cycle engines are still at low to mid-levels of development, while chemical rocket engines are reaching their theoretical peak efficiency.

The use of recoverable first stages for space launchers requires the analysis of flight paths that the vehicle can follow to be recovered for refurbishment and future reuse. Due to the limited available locations that allow a safe horizontal landing in an airfield or spaceport, as well as the safety and regulatory requirements, planning for recovery and abort scenarios is a critical task that has to be included from a very early stage in the vehicle design process.

The vehicle considered in this study is a lifting body vehicle powered by a combined cycle engine. The performances of the vehicle are studied with a software ${ }^{1-3}$ for trajectory and multidisciplinary design optimisation (MDO) that is extremely modular and can change the mathematical models to cater for different needs of model accuracy or computational runtime depending on the requirements.

An initial study is performed to determine the best ascent profile under nominal conditions by optimising the trajectory of the vehicles to deliver the maximum payload to a target orbit. Abort scenarios are simulated at different points along the ascent trajectory by removing the propulsion (turning off the engine but keeping the fuel onboard) and optimising the glide descent path. The re-entry flight is optimised to find the maximum range that the vehicle can reach in both downrange and crossrange distance.

\section{System models}

This section describes the mathematical models used to simulate the vehicle performance over the mission. Specifically, models are presented for 3DOF flight dynamics, open loop control of the thrust and vehicle attitude, propulsion system, vehicle aerodynamics, environment models for the Earth's geometry, atmosphere and gravity.

*PhD Student, federico.toso@strath.ac.uk, AIAA Student Member.

${ }^{\dagger}$ Lecturer of Aerospace Engineering, AIAA Member. 


\section{A. Flight dynamics and control}

The vehicles are modelled as a point mass in an Earth Centered Earth Fixed reference frame with 3 degrees of freedom for all phases of the mission. The state vector $\mathbf{x}=[h, \lambda, \theta, v, \gamma, \chi]$ contains the 6 variables for position and velocity, explicitly defined as altitude $h$, velocity $v$ relative to the reference frame, flight path angle $\gamma$ measured from the local horizon, heading angle $\chi$ defined clockwise from the local North, and $\lambda$ and $\theta$ as latitude and longitude coordinates on the Earth. The derivatives of the state variables are calculated with,

$$
\begin{aligned}
\dot{h}= & \dot{r}=v \sin \gamma \\
\dot{\lambda} & =\frac{v \cos \gamma \sin \chi}{r} \\
\dot{\theta}= & \frac{v \cos \gamma \cos \chi}{r \cos \lambda} \\
\dot{v}= & \frac{F_{T} \cos \alpha-D}{m}-g_{r} \sin \gamma+g_{\phi} \cos \gamma \cos \chi+\omega_{\mathrm{E}}^{2} r \cos \lambda(\sin \gamma \cos \lambda-\cos \gamma \sin \chi \sin \lambda) \\
\dot{\gamma}= & \frac{F_{T} \sin \alpha \cos \mu+L}{m v}-\frac{g_{r}}{v} \cos \gamma-\frac{g_{\phi}}{v} \sin \gamma \cos \chi+\frac{v}{r} \cos \gamma+2 \omega_{\mathrm{E}} \cos \chi \cos \lambda \\
& \quad+\omega_{\mathrm{E}}^{2}\left(\frac{r}{v}\right) \cos \lambda(\sin \chi \sin \gamma \sin \lambda+\cos \gamma \cos \lambda) \\
\dot{\chi}= & \frac{L \sin \mu}{m v \cos \gamma}-g_{\phi} \sin \chi-\left(\frac{v}{r}\right) \cos \gamma \cos \chi \tan \lambda+2 \omega_{\mathrm{E}}(\sin \chi \cos \lambda \tan \gamma-\sin \lambda) \\
& \quad-\omega_{\mathrm{E}}^{2}\left(\frac{r}{v \cos \gamma}\right) \cos \lambda \sin \gamma \cos \chi
\end{aligned}
$$

where $D$ and $L$ are the aerodynamic lift and drag forces, $m(t)$ is the mass of the vehicle and $\omega_{\mathrm{E}}$ is Earth's rotational speed. The gravity is decomposed in two components of the force, the tangential $g_{\phi}$ and the radial $g_{r}$. The vehicle controls are the direction and magnitude of the thrust vector $F_{T}$ through the modulation of the throttle, the angle of attack $\alpha$ and bank angle $\mu$ of the vehicle. The propulsion system is non-gimbled; the thrust vector produced is aligned with the longitudinal body axis of the vehicle.

The coordinates are converted into the Earth Centered Inertial frame to compute the Keplerian orbital parameters for the semimajor axis $a$, eccentricity $e$ and inclination $i$.

\section{B. Environment}

The WGS84 geodetic system is used to model the Earth geometric parameters including the radius length from the centre to the Earth surface $r_{/} E$ as a function of latitude. The rotation of the planet is $\omega_{\mathrm{E}}=$ $7.292115 e-5 \mathrm{rad} / \mathrm{s}$.

The gravitation model is based on spherical harmonics accounting for the $J_{2}, J_{3}$ and $J_{4}$ terms. The radial and tangential components of the gravitational pull are computed from the local radius $r_{E}$, the vehicle's height above surface $h$ and the latitude $\lambda$.

The atmosphere is modelled using the static global International Standard Atmosphere. The model is expanded above the maximum altitude $h_{\max }=84852 \mathrm{~m}$ by extending the exponential laws regulating pressure and density assuming constant temperature $T\left(h>h_{\max }\right)=186.87 \mathrm{~K}$.

\section{Vehicle configuration}

The vehicle is a horizontal take off and landing, reusable launch vehicle originally designed for a target mission of releasing $1000 \mathrm{~kg}$ of payload in a $h=200 \mathrm{~km}$ circular orbit from a high latitude spaceport. The gross and dry mass values are obtained from the multidisciplinary optimisation ${ }^{3}$ of a vehicle performed using the lift and drag coefficients for a conceptual hypersonic test vehicle Lazarus, ${ }^{4}$ a conceptual study focused on the development of aerodynamic laws for the purpose of scaling surfaces. This results in a vehicle with an optimised gross take off mass $m_{\text {gtow }}=90035 \mathrm{~kg}$ and a dry mass of $m_{d r y}=22907 \mathrm{~kg}$, equal to an inert mass margin $m_{\text {dry }} / m_{\text {gtow }}=0.254$.

The Lazarus vehicle was designed as an SSTO with ejector scram-rocket engines which require a large $2 \mathrm{D}$ inlet. As the propulsion system for this vehicle assumes a different type of rocket-based combine cycle 
engine with reduced inlet area, the aerodynamics were recalibrated based on experimental wind tunnel test results for the $\mathrm{X}-34 .^{5}$ A design optimisation aimed at sizing the propulsion system is performed concurrently with the ascent trajectory analysis.

\section{Aerodynamics}

The database ${ }^{5}$ of aerodynamic characteristics resulted from the studies of the X-34 vehicle presents an extended set of coefficients $C_{L}$ and $C_{D}$ values gathered from wind tunnel tests. The data points cover a set of inputs in a range of Mach number $0.4 \leq M \leq 6$ and angle of attack $-4 d e g \leq \alpha \leq 24 d e g$, while the Lazarus model is valid only in the supersonic regime. The database is used to create two functions $C_{L}=f(\alpha, M)$ and $C_{D}=f(\alpha, M)$ obtained through polynomial interpolation. The order of the polynomial is the lowest that guarantees a coefficient of determination $R \geq 0.99$, assuring accurate results and fast computation. The $R$ value is guaranteed inside the domain of the experimental test results for the X-34, therefore the controls of the vehicle are bound within the limits of angle of attack and Mach of the dataset. Whenever the Mach is outside of the experimental points range, the value is treated as equal to the closest bound in the interpolating equation. The reference aerodynamic surface of the vehicle is calculated by choosing the same take-off aerodynamic load of the X-34 test vehicle of $W / S_{r e f}=2423 \mathrm{~N} / \mathrm{m}^{2}$, resulting in an area $S_{\text {ref }}=364.64 \mathrm{~m}^{2}$.

The upper stage configuration is an expendable rocket with a cylindrical body, no lifting surfaces. The aerodynamic model uses a constant drag coefficient $C_{D}=1$, lift coefficient $C_{L}=0$ and a reference surface area $S_{\text {ref }}=1 \mathrm{~m}^{2}$.

\section{E. Propulsion}

The first stage propulsion system is a hydrogen fuelled motor capable of functioning as both an air breathing engine, using atmospheric oxygen, and rocket, using onboard LOX at the catalyst. The use of a high-speed air breathing engine is the crucial requirement to reach a suitable LEO/LEO-injection altitude at a high velocity without a second stage. This can be achieved by a number of differing hybrid propulsion systems, such as ejector-ramjet-scramjet rocket combinations to the more integrated Synergistic Air-Breathing Rocket Engine (SABRE) under development by Reaction Engines. ${ }^{6}$ The engine performance here is modelled based on the published performance specifications for the SABRE.

The rocket maximum vacuum a specific impulse is $I_{s p}=450 \mathrm{~s}$, modelled using the general rocket equation accounting for the nozzle expansion losses due to changes in atmospheric pressure.

At lower altitudes, generally below $30 \mathrm{~km}$, the engine can exploit the presence of oxygen in the atmosphere and it is modelled as a modified rocket engine with variable $I_{s p}$ and performance depending on the flight conditions. The maximum equivalent specific impulse is $I_{s p}=6500 \mathrm{~s}$. Increasing altitude negatively affects this value due to the decrease of air pressure and density. Quadratic laws were developed that reach the peak efficiency value at $M=3.5$ and $100 \%$ throttle to model this phenomenon. The thrust is computed with the equation $T=m_{p} g_{0} I_{s p}$.

The switching point between the two engine operational modes is unconstrained and is a result of the optimisation of the ascent.

The upper stage is modelled as a small hydrazine thruster, with $I_{s p}=250 \mathrm{~s}$, and the same formulation used for the nozzle losses of the first stage rocket.

The propellant mass flows for both stages and all engine operating modes are optimisation variables in the trade off analysis of the ascent. The descent cases analyses are set up as unpowered glides by imposing a zero throttle along the whole trajectory.

\section{Optimisation}

The trajectory of the vehicle is decomposed in user defined phases characterised by different system models and problem formulations. This allows the fusion of approaches that may otherwise present mathematical discontinuities if coupled and evaluated together such as diverse control strategies, changes in engine operating mode or staging. The optimisation algorithm of choice is the gradient based solver sequential quadratic programming of MATLAB's function fmincon. To further increase the exploration capabilities and chances of convergence of the function, a multistart approach is used with relaxed constraints and coarser timestep. 
The solution found is used as a first guess if multiple cases with similar starting conditions are analysed, such as the descent ones in the following sections.

\section{A. Optimal control problem formulation}

The optimal control problem is formulated with a direct transcription, multiple shooting method. The trajectory is divided in $n_{P}$ user defined phases with different system models, control bounds or coarser/finer meshes. Every phase is divided into $n_{E}$ segments with $n_{C}$ discrete control nodes. The nodes define the control grid that is analysed during the trajectory propagation to determine the attitude of the vehicle. In this analysis the Chebyshev nodes are used and the values between the nodes are computed with a Piecewise Cubic Hermite Interpolating Polynomial. Each of the multiple phases is set up with the specific set of mathematical models and, in this case, numerically integrated with a $4^{\text {th }}$ order fixed timestep Runge Kutta method following the control law.

The optimisation vector is constructed element by element, each of those blocks containing the values of the controls in the nodes, the initial state variables and the duration of the trajectory segment. The states and controls are matched between elements to guarantee a continuous flight. The values at end of a segment resulted from a trajectory propagation have to be equal to the ones at the beginning of the next segment. Additional equality constraints are set on the final values of the flight to reach desired conditions such as a predetermined orbit and to assure a correct mass separation when staging. Inequality constraints include acceleration and dynamic pressure limits. Inequality is also used if the final state values have to be greater or less than a defined amount. The tolerance on all constraints is $10^{-4}$.

The algorithm used is the single objective sequential quadratic programming. The cost functions used for this analysis are the maximum payload, maximum downrange and crossrange distances.

\section{B. First guess generation}

To identify a suitable first guess, a pool of starting guesses from the search space is generated with Latin Hypercube Sampling and run through the optimisation routine with relaxed constraints and 3 times longer timestep, reducing the tolerance on both the equality and inequality ones to $10^{-3}$. This approach increases the exploration capabilities of the algorithm. The converged solution with best cost function is used for the analysed data presented.

This approach to find a solution closer to the global minimum is computationally expensive and well suited for single case analysis. In the study presented for the abort scenarios multiple points have to be evaluated, therefore the time and resources required to complete the calculations with such approach are prohibitive. The converged solutions are passed as 'warm start' initial guesses to a second optimisation routine set with the original tolerances and timestep length.

Choosing closely spaced abort points means that the starting conditions of consecutive cases have minor differences and the solution for the previous starting point can be used to provide the optimisation routine with a good starting guess that can quickly converge to a solution and repeat the iterative process, sampling the whole search space only once.

\section{Test cases and results}

\section{A. Nominal ascent trajectory}

The nominal ascent mission is the deployment of $m_{p}=1000 \mathrm{~kg}$ of payload in a $200 \mathrm{~km}$ circular orbit. The chosen starting location for the ascent at $\left(0^{\circ} \mathrm{N}, 0^{\circ} \mathrm{E}\right)$ latitude and longitude. Placing the origin of the axis in this location does not require reference frame transformations to compute distances. The same approach can be applied to any other starting point on Earth.

The aerodynamic model introduced requires a new evaluation of the optimal value of maximum thrust to reach the imposed objective. Two engine scaling parameters are added to the optimisation vector, one per stage. The first stage can scale the thrust levels of the air breathing mode in the range [2.5, 4.8] MN, while the rocket vacuum maximum thrust is in the range $[3.2,6] \mathrm{MN}$. The second stage boundaries are $[500,5000] \mathrm{N}$.

The starting conditions of the ascent are $h_{0}=1000 \mathrm{~m}, v_{0}=150 \mathrm{~m} / \mathrm{s}, \gamma_{0}=0 \mathrm{deg}, \theta_{0}=90 \mathrm{deg}, \lambda_{0}=0$ deg, $\chi_{0}=0 \mathrm{deg}, m_{0}=90035 \mathrm{~kg}$. The controls used for the equatorial prograde ascent are the angle of attack 
$-4 \operatorname{deg} \leq \alpha \leq 24 \mathrm{deg}$ and the throttle $0 \leq \tau \leq 1$. The upper stage has an initial mass $m_{0}=1000 \mathrm{~kg}$ and the initial starting conditions are part of the optimisation variables. The controls for the upper stage increase the range of available angle of attack to $-30 \mathrm{deg} \leq \alpha \leq 30 \mathrm{deg}$. The starting values of angle of attack and roll are constrained to be the same of the first stage at the instant of release, while the throttle is not.

The choice of starting the ascent from the intersection of Greenwich meridian and the Equator and eastward flight simplifies the visualization of the results; the impact of latitude of launch site and inclination of the target orbit has been extensively documented and analysed in past studies. ${ }^{3}$ The cost function to maximise in the ascent optimisation is the final payload mass.

The first stage flight is divided in two phases of $n_{e}=3$ multiple shooting elements, each with $n_{c}=5$ control points. The second stage has the task of circularizing the orbit by raising the perigee if the first stage cannot. A reduced number of elements $n_{e}=2$ is used in this phase, while the number of control points per element remains constant.

Table 1: Upper and lower bounds for ascent analysis

\begin{tabular}{rlll}
\hline & $\begin{array}{l}\text { Phase 1 } \\
(\text { Stage 1, Air-breathing })\end{array}$ & $\begin{array}{l}\text { Phase 2 } \\
(\text { Stage 1, Rocket })\end{array}$ & $\begin{array}{l}\text { Phase 3 } \\
\text { (Stage 2, Rocket) }\end{array}$ \\
\hline Altitude $h, \mathrm{~km}$ & {$[1,100]$} & {$[10,200]$} & {$[10,200]$} \\
Velocity $v, \mathrm{~m} / \mathrm{s}$ & {$[100,2500]$} & {$[1000,800]$} & {$[1000,8000]$} \\
Flight path angle $\gamma, \operatorname{deg}$ & {$[-60,+90]$} & {$[-60,+90]$} & {$[-60,+90]$} \\
Heading angle $\chi, \operatorname{deg}$ & {$[-180,+180]$} & {$[-180,+180]$} & {$[-180,+180]$} \\
Latitude $\lambda, \operatorname{deg}$ & {$[-90,+90]$} & {$[-90,+90]$} & {$[-90,+90]$} \\
Longitude $\theta, \operatorname{deg}$ & {$[-180,+180]$} & {$[22907,90035]$} & {$[-180,+180]$} \\
Mass $m, \mathrm{~kg}$ & {$[22907,90035]$} & {$[-4,24]$} & {$[-30,30]$} \\
\hline Angle of attack $\alpha, \operatorname{deg}$ & {$[-4,24]$} & {$[0,1]$} & {$[0,1]$} \\
Throttle $\tau$ & {$[0,1]$} &
\end{tabular}

The optimisation results in a final mass $m_{p}=999.933 \mathrm{~kg}$ delivered to the target orbit. The mass lost by the second stage is due to the very short circularization burn performed at the apogee. The optimised value of upper stage thrust $T_{2}=500 \mathrm{~N}$ is on the lower bound. The reusable stage maximum thrust values for the airbreathing and rocket mode are respectively $T_{S L, \text { max }}=3.894 \mathrm{MN}$ and $T_{v a c, \text { max }}=4.984 \mathrm{MN}$. From the throttle profile emerges the exploitation by the optimisation routine of the often used strategy of coasting to the apogee to raise the perigee.

\section{B. Descent case analysis}

The analysis of the abort trajectories uses the results generated from the ascent optimisation as starting point for the descent abort scenarios. The ascent throttle profile shows the separation between an initial powered phase in the air-breathing and rocket segments followed by a ballistic flight. During this phase, the vehicle will coasts till the apogee of the suborbital arc, where a final boost circularizes the orbit. This phenomena simplifies the problem allowing the study of the abort trajectories in the first 4 elements of the trajectory covering the main powered part of the ascent. After the engine cut off point the trajectory would closely follow a nominal ballistic suborbital arc followed by a final glide re-entry. With no thrust, no extra energy is introduced into the system. Studying any of the points after the end of the $4^{\text {th }}$ element would yield similar results. A set of 50 data points is selected to guarantee a fine grid of starting points without being too computationally intensive. Those cover a set of altitudes $1 \mathrm{~km}<h_{0}<108 \mathrm{~km}$ and velocities $271 \mathrm{~m} / \mathrm{s}$ $<v_{0}<6327 \mathrm{~m} / \mathrm{s}$. The points are equally spaced in time every 6 seconds along the ascent trajectory. The states variables corresponding to those selected points are used as starting conditions for each of the abort scenarios.

The descent phase is constrained to achieve a final state corresponding to a landing approach with an altitude $h_{f}=1000 \mathrm{~m}$, speed $v_{f}=150 \mathrm{~m} / \mathrm{s}$ and flight path angle $\gamma<10^{\circ}$. Given the assumption of equatorial ascent, it is reasonable to use only the longitudinal control while maximizing the descent range. The angle of 
attack of the vehicle is therefore constrained between the limits of the aerodynamic model $-4^{\circ} \leq \alpha \leq 24^{\circ}$, while throttle and bank angle are fixed to $\tau=0^{\circ}$ and $\mu=0^{\circ}$.

The glide descent is composed of a single phase subdivided in $n_{e}=3$ multiple shooting segments, each containing $n_{c}=7$ control points following a Chebyshev nodes distribution. The values of control between the points are obtained with a piecewise cubic Hermite spline. The integration of the trajectory is performed with a $4^{\text {th }}$ order RungeKutta method with fixed timestep. Constraints limit the peak accelerations and dynamic pressure respectively to $\left|a_{x, z}\right| \leq 6 g_{0}$ and $\max (Q) \leq 20 \mathrm{kPa}$. The distance from the starting abort point, evaluated with the Haversine formula, is the maximised optimisation metric.

Table 2: Upper and lower bounds for the descent downrange and crossrange analyses

\begin{aligned} & \hline Altitude, km ${[1,200] } \\ &$ Velocity, m/s ${[150,8000] } \\ &$ Flight path angle, deg ${[-60,+90] } \\ &$ Heading angle, deg ${[-180,+180] } \\ &$ Latitude, deg ${[-90,+90] } \\ &$ Longitude, deg ${[-180,+180] } \\ &$ Mass, kg ${[22907,90035] } \\ &$\hline Angle of attack $\alpha, \operatorname{deg} {[-4,24] } \\ &$ Bank angle $\mu, \operatorname{deg} {[-80,80] } \\ &$\hline\end{aligned}

A further analysis is subsequently performed by adding the bank angle control $-80 \operatorname{deg} \leq \mu \leq 80$ deg starting from the same abort points to identify the crossrange capabilities of the vehicle. The bank angle rotates the lift vector around the velocity axis. The single phase of the downrange analysis is separated into two, the first one with 2 elements has the additional control of the bank angle. The last phase, made of a single element, remains with only the control of the angle of attack. This choice has been made because during the first trial runs a faulty behaviour emerged: The long duration of the reentry from higher starting energies and the coarse control mesh chosen made the vehicle enter spirals that reduced altitude without gain in crossrange capabilities. The chosen setup eliminates the problem and does not require an increased node number. In this case the optimisation metric is the maximum latitude. The total crossrange is obtained by calculating the arc length from the equator to the final latitude.

\section{Maximum downrange results}

Out of the 50 points analysed, five points are chosen as representative of the group of solutions. The first four (A, B, C, E) are selected at the end of the respective ascent trajectory elements. A $5^{\text {th }}$ one (D) is picked in the middle of the rocket flight segment. Table 3 lists the initial state vector for each point. The results for the downrange analysis are shown in Figs 2.

Table 3: Starting conditions of the selected result points highlighted in the descent graphs

\begin{tabular}{crrrrrrr}
\hline Point & Altitude $[\mathrm{m}]$ & Velocity [m/s] & FPA [rad] & Heading [rad] & Lat [rad] & Lon [rad] & Mass [kg] \\
\hline A & 4201 & 339.4 & 1.0417 & 1.5708 & 0 & 0.0009 & 89056 \\
B & 23879 & 1272.5 & 0.1151 & 1.5708 & 0 & 0.0064 & 85578 \\
C & 55899 & 2351.5 & 0.1210 & 1.5708 & 0 & 0.0382 & 78099 \\
D & 75048 & 4644.2 & 0.1039 & 1.5708 & 0 & 0.0668 & 45531 \\
E & 107650 & 6327.9 & 0.1009 & 1.5708 & 0 & 0.1142 & 30638 \\
\hline
\end{tabular}

The energy plot in Figure 3 presents the maximal downrange distance that the suborbital flights can reach from the starting abort points. The curve outlines the front of solutions found with the approach described. The small areas where the line does not follow the trend are due to the flight path angle excursion 
of the starting conditions. From the same values of altitude and velocity, the direction of the latter impacts the performances.

While the skip re-entry is a return strategy that has been used by many re-entry vehicles, it does present practical issues such as added stress on the structure, limitations on the lifetime of the vehicle (reusuability), onboard control and stability, etc. The phugoids can be mitigated within the analysis through more strict acclerations/loading limits, h further constraints or post-processing of the controls though this would represent a suboptimal result considering only the maximum range possible. The focus of this study was to evaluate the maximum capability of the vehicle under emergency situations.

\section{Maximum cross range results}

Figures 4 show the time histories of controls and states in the descent case. It can be seen that the case with bank show the lack of significant aerodynamic forces in the high altitude suborbital arcs. The relation between crossrange and starting energy is shown in Figure 5.

The combined locations of the coordinates of the final points are shown in Figure 6 on the latitudelongitude plane. The five sample points are highlighted to clearly show the distribution of solutions and therefore maximum range of glide flight of the vehicle.

\section{Conclusion}

The downrange and crossrange distance performances in case of abort events during the ascent of a spaceplane have been studied. The results obtained can be applied to the study of the abort procedures from any launch site.

The first guess creation through multistart analysis on the first point and successive iterative generation approach has succeeded in providing results for all the abort points analysed. This technique reached convergence in all cases, eliminating the need of repeated explorative analysis, saving computational time. The energy-distance plots can be a useful tool to study trade-offs between vehicles, launch locations, abort procedures and risk assessment. The algorithm can also be used perform a MDO to satisfy regulatory or technological constraints.

\section{References}

\footnotetext{
${ }^{1}$ Pescetelli, F., Minisci, E., Maddock, C., Taylor, I., and Brown, R., "Ascent Trajectory Optimisation for a Single-Stageto-Orbit Vehicle with Hybrid Propulsion," AIAA/3AF International Space Planes and Hypersonic Systems and Technologies Conference, 2012.

${ }^{2}$ Toso, F., Maddock, C., and Minisci, E., "Optimisation of Ascent Trajectories for Lifting Body Space Access Vehicles," Space transportation solutions and innovations symposium, International Astronautical Congress, 2015.

${ }^{3}$ Toso, F. and Maddock, C., "Deployed payload analysis for a single stage to orbit spaceplanes," Space transportation solutions and innovations symposium, International Astronautical Congress, 2016.

${ }^{4}$ Young, D. A., Kokan, T., Clark, I., Tanner, C., and Wilhite, A., "Lazarus: A SSTO Hypersonic Vehicle Concept Utilizing RBCC and HEDM Propulsion Technologies," International Space Planes and Hypersonic Systems and Technologies Conference, AIAA, 2006.

${ }^{5}$ Brauckmann, G. J., "X-34 vehicle aerodynamic characteristics," Journal of spacecraft and rockets, Vol. 36, No. 2, 1999, pp. 229-239.

${ }^{6}$ Hempsell, M., Longstaff, R., and Bond, A., "SKYLON Users Manual," Reaction Engines Limited, Vol. 1, 2010.
} 


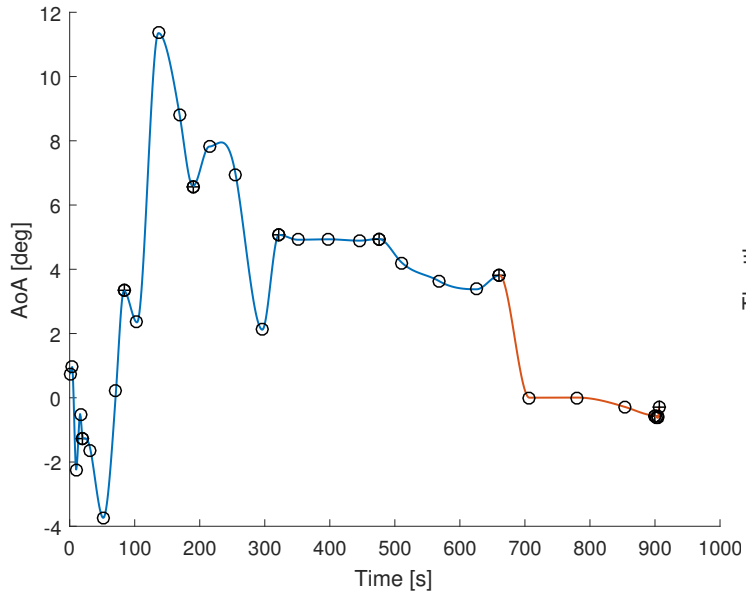

(a) Angle of attack

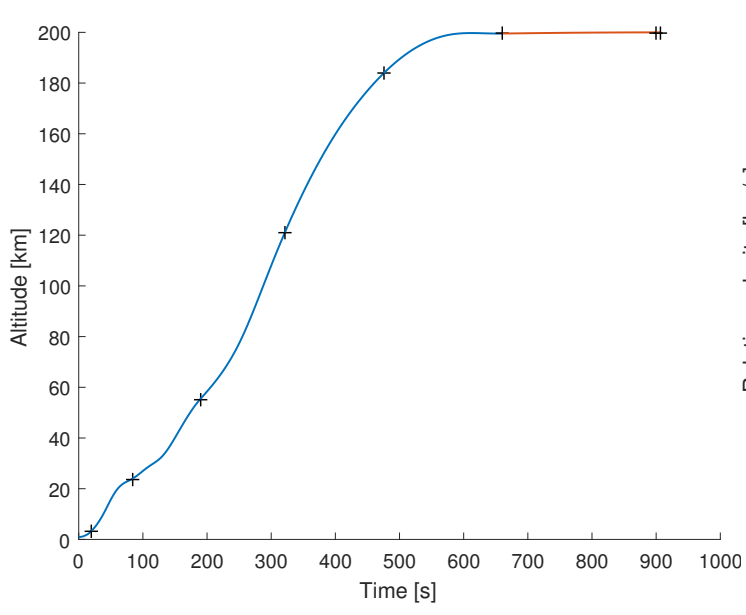

(c) Altitude as a function of time

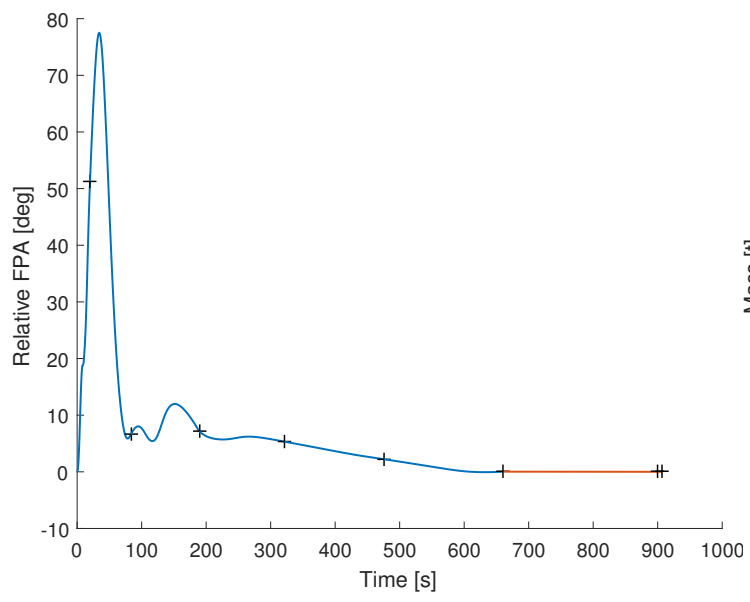

(e) Flight path angle

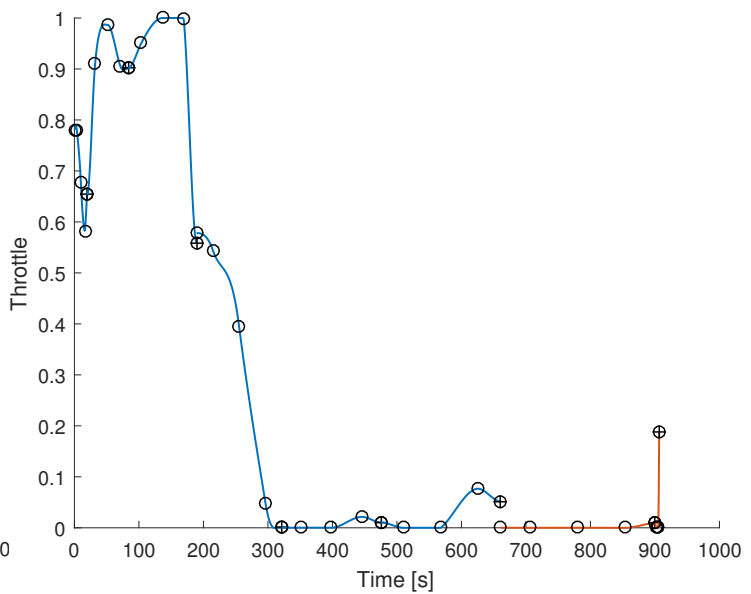

(b) Throttle control

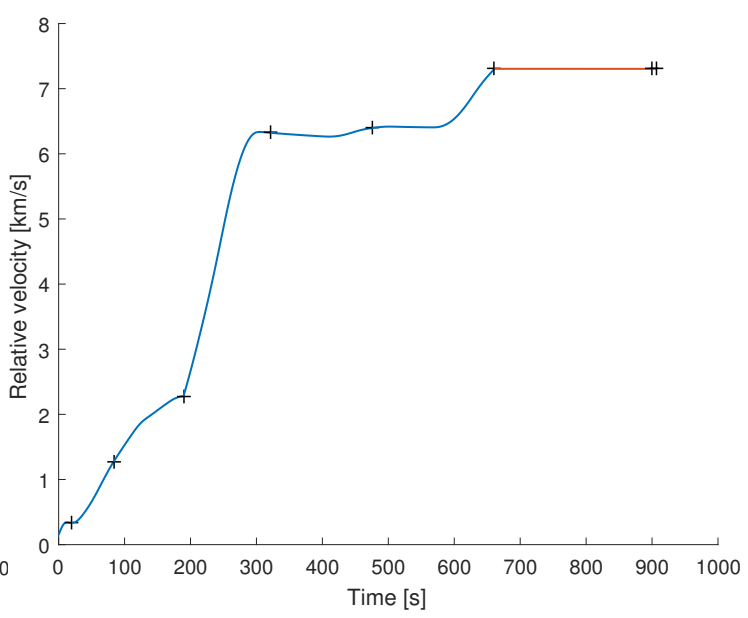

(d) Velocity

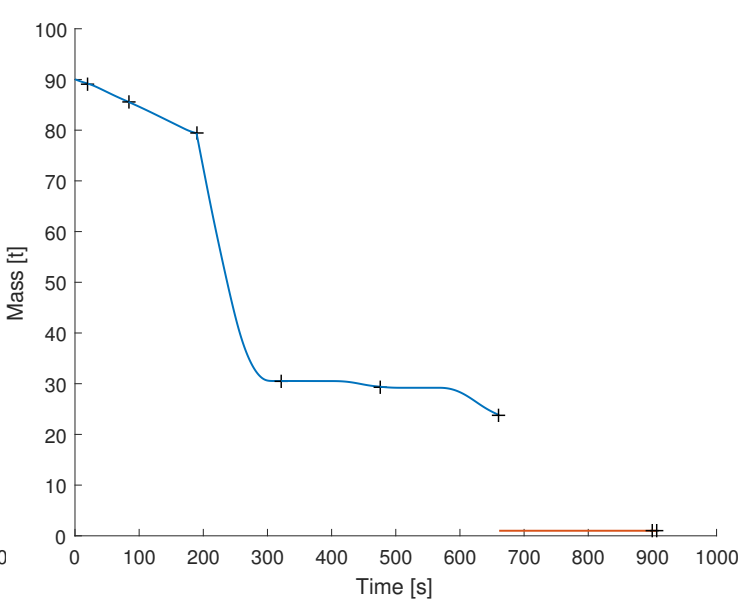

(f) Vehicle mass

Figure 1: Time histories for controls and states of the ascent case solution. Cross markers are placed at the element junction points. Circle markers highlight the values of control nodes. The trajectory is coloured in blue for the first stage, orange for the second one. The end of the airbreathing phase coincides with the third cross marker. 


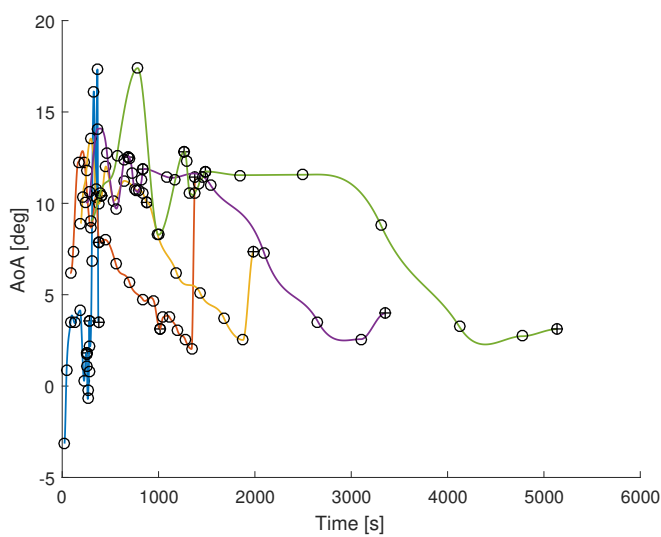

(a) Angle of attack

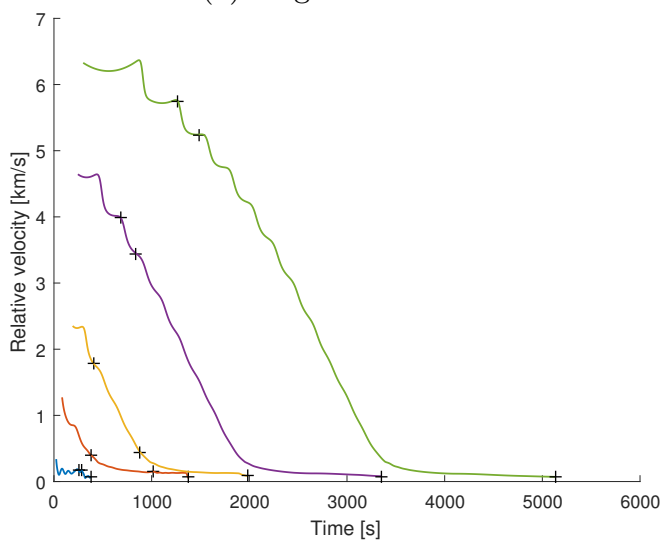

(c) Velocity

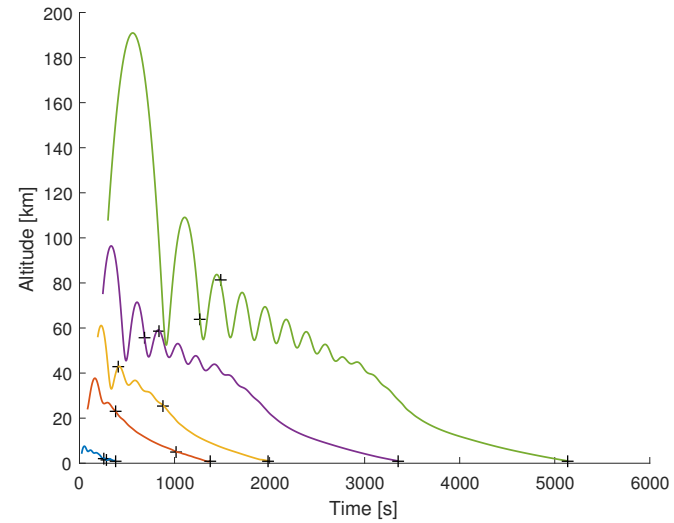

(b) Altitude as a function of time

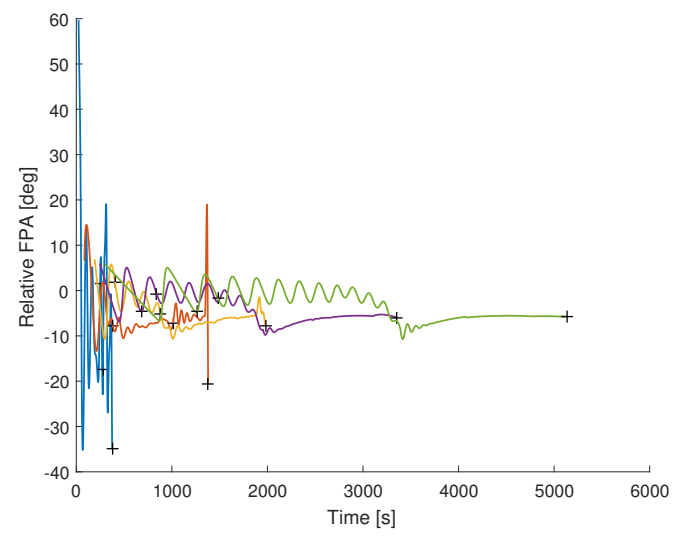

(d) Flight path angle

Figure 2: Time histories for controls and states of the descent case without bank. Cross markers are placed at the element junction points. Circle markers highlight the values of control nodes

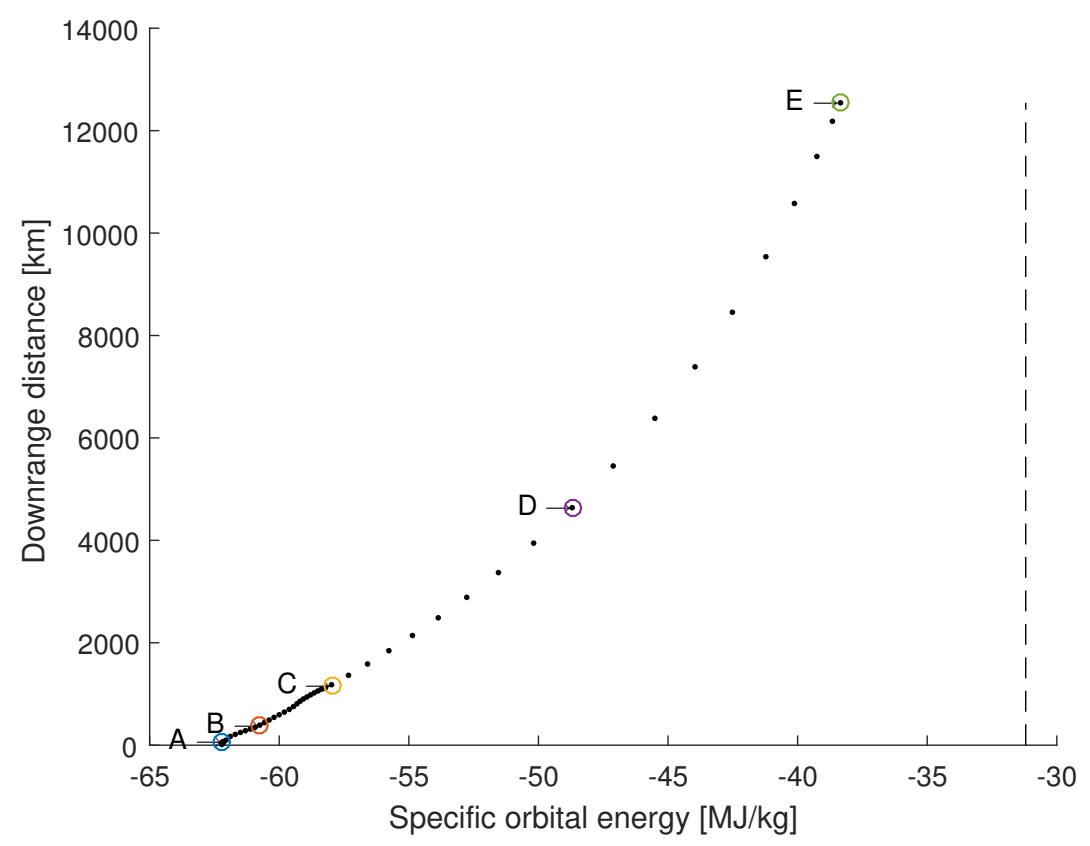

Figure 3: Relationship between starting specific orbital energy and downrange distance. The dashed line is the minimal energy required for a stable orbit around the Earth. 


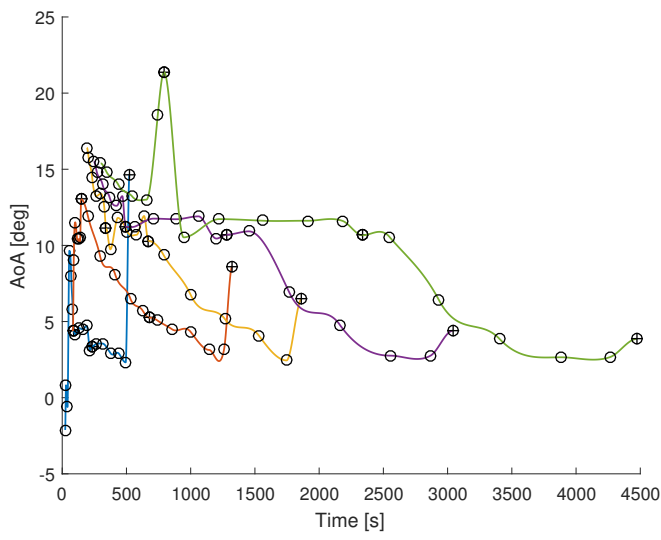

(a) Angle of attack

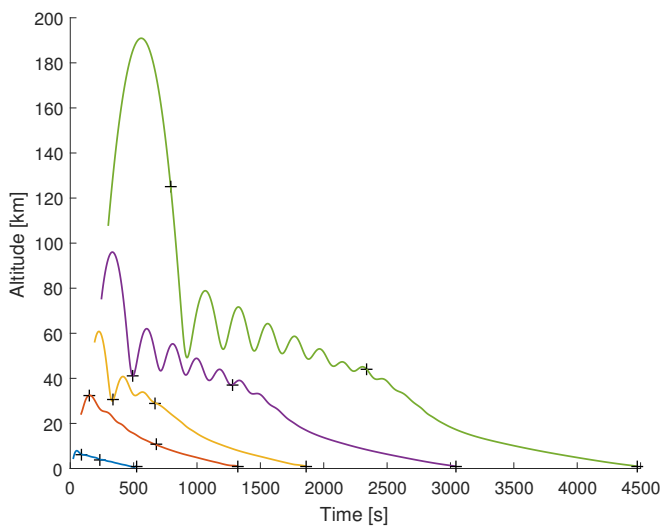

(c) Altitude as a function of time

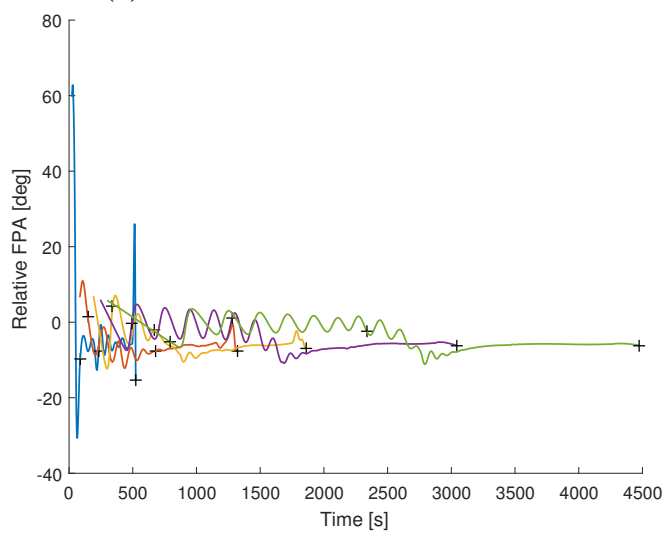

(e) Flight path angle

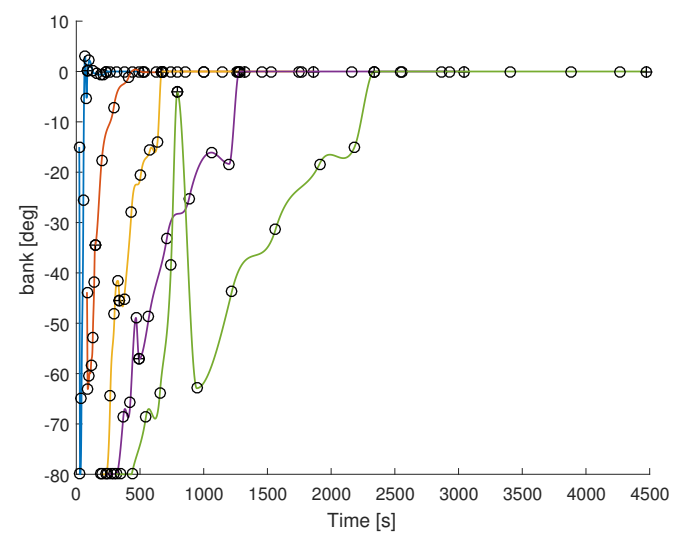

(b) Bank angle

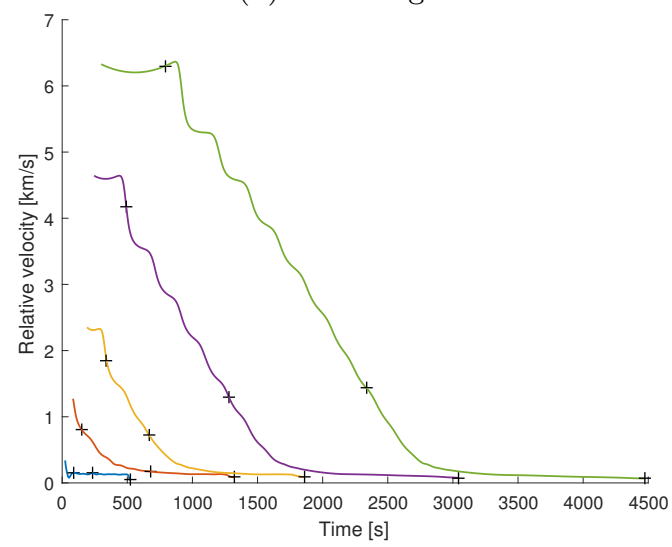

(d) Velocity

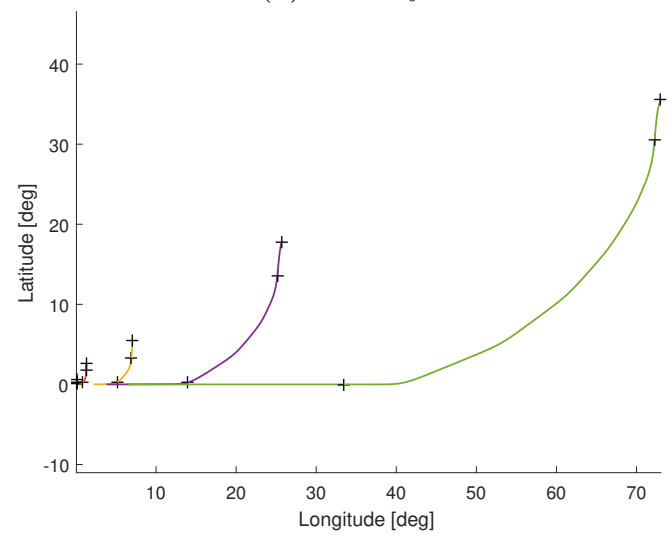

(f) latitude

Figure 4: Time histories for controls and states of the descent case with bank. Cross markers are placed at the element junction points. Circle markers highlight the values of control nodes. 


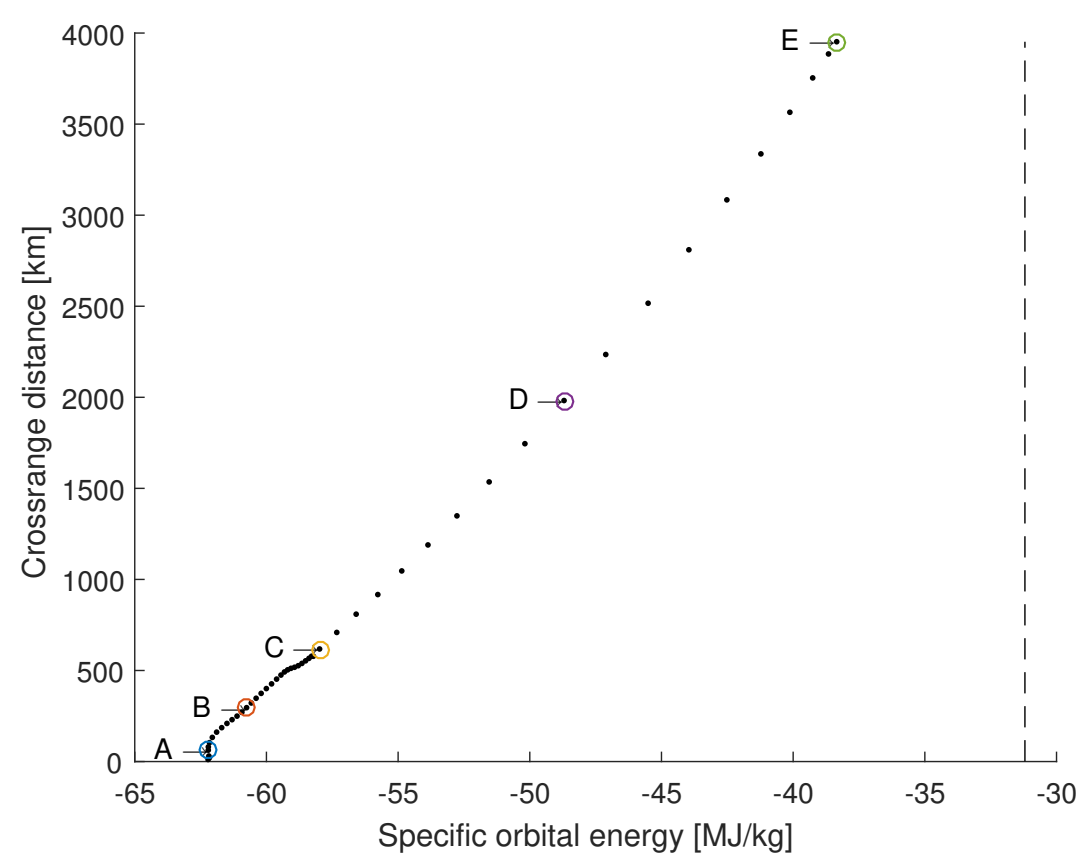

Figure 5: Relationship between starting specific orbital energy and crossrange distance. The dashed line is is the minimal energy required for a stable orbit around the Earth.

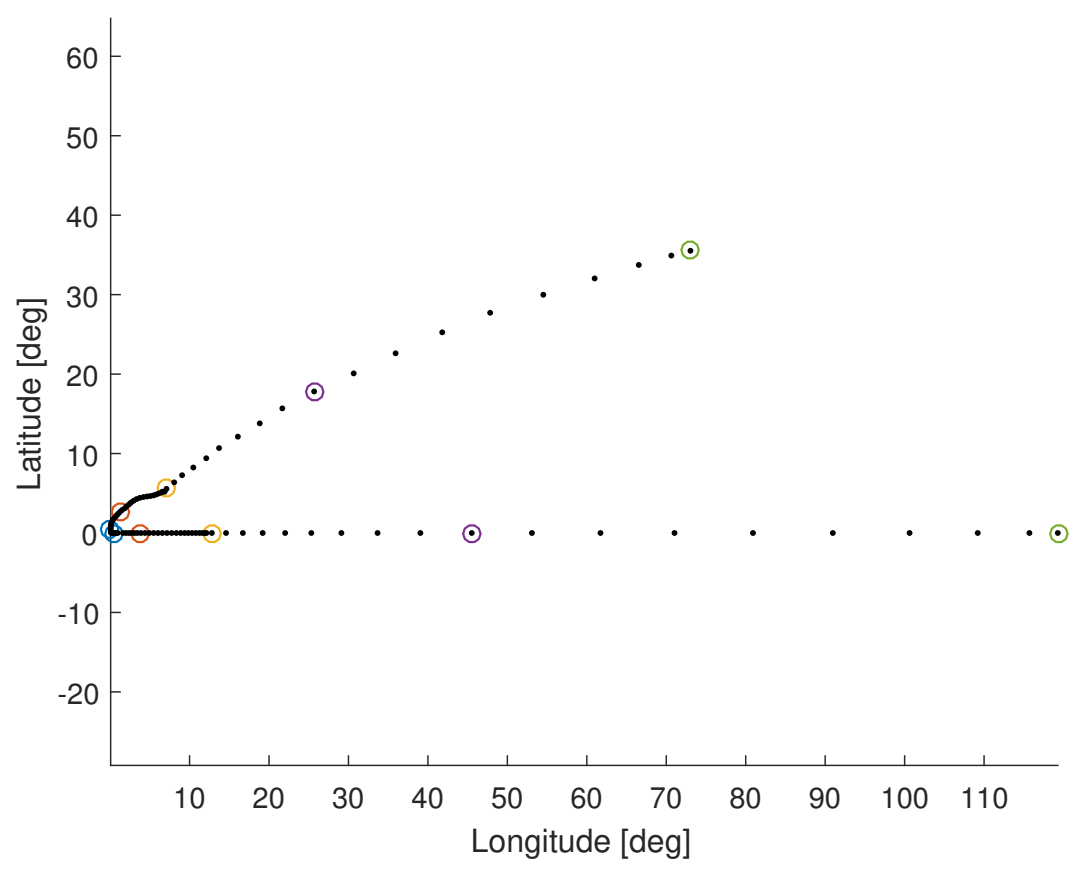

Figure 6: Latitude-Longitude distribution of the final points of descents for the analyses with and without bank 